\title{
Multicomponent Skyrmion lattices and their excitations
}

\author{
D.L. Kovrizhin ${ }^{1,2}$, Benoît Douçot ${ }^{3}$, and R. Moessner ${ }^{1}$ \\ ${ }^{1}$ Max Planck Institute for the Physics of Complex Systems, Nöthnitzer Str. 38, 01187 Dresden, Germany \\ ${ }^{2}$ Russian Research Centre, Kurchatov institute, 1 Kurchatov sq., 123098, Moscow, Russia and \\ ${ }^{3}$ LPTHE, Université Pierre et Marie Curie-Paris 6 and CNRS UMR 7589, Boite 126, 4 Place Jussieu, 75252 Paris Cedex 05
}

\begin{abstract}
We study quantum Hall ferromagnets with a finite density topologically charged spin textures in the presence of internal degrees of freedom such as spin, valley, or layer indices, so that the system is parametrised by a $d$-component complex spinor field. In the absence of anisotropies, we find formation of a hexagonal Skyrmion lattice which completely breaks the underlying $S U(d)$ symmetry. The ground state charge density modulation, which inevitably exists in these lattices, vanishes exponentially in $d$. We compute analytically the complete lowlying excitation spectrum, which separates into $d^{2}-1$ gapless acoustic magnetic modes and a magnetophonon. We discuss the role of effective mass anisotropy for $S U(3)$-valley Skyrmions relevant for experiments with AlAs quantum wells. Here, we find a transition, which breaks a six-fold rotational symmetry of a triangular lattice, followed by a formation of a square lattice at large values of anisotropy strength.
\end{abstract}

PACS numbers: 73.43.-f, 71.10.-w, 73.43.Lp, 73.21.-b 81.05.Uw

Introduction. Skyrmions in quantum Hall ferromagnets [1] present an early example of topological excitations in condensed matter physics, a topic of persistently large interest, which by now has grown to encompass itinerant magnets with spin-orbit coupling [2, 3] and spinor condensates [4]. In the quantum Hall effect (QHE), these topological textures carry a quantised charge, endowing them with stability and providing possible probes via Coulomb interactions and charge transport. The low-energy modes of these defects have possibly been observed in NMR measurements [5]. The textures are also exceptionally tunable, as their density, and hence the relative strength of interactions, can be modified by a gate potential or magnetic field. The possibility of controlling spin by coupling to electron charge makes these systems interesting candidates for spintronics applications.

In this work, we discuss the physics of Skyrmions with an enlarged internal space. This space minimally consists of two components which are, most simply, represented by physical up and down spins - but other degrees of freedom frequently play a role. Early examples are the layer index in double quantum wells of GaAs, where interlayer phase coherence spontaneously develops [6, 7], or the valley degeneracy in semiconductors [8]. Recently, there has been a further proliferation, in the form of spin, layer and valley degeneracies in graphene [9], and cold atoms [4], where $S U(N)$ internal degrees of freedom and Skyrmions have emerged as interesting topics.

At finite density of defects and in presence of Coulomb interactions, Skyrmions can form a crystal (SC), analogous to Skyrme crystals of nuclear physics [10]. The properties of SCs have been extensively discussed in the $S U(2)$ case, and a large number of results in a wide variety of settings have been obtained using Hartree-Fock (HF) theory for $S U(4)$ systems [11]. Most of these studies are based on the numerical solution of the HF equations of motion, being hard to perform due to presence of several lengthscales.

This motivates us to develop an analytical approach to the general case of Skyrmion crystals with an internal space parametrised locally by a $d$-component complex spinor. The simplifying feature of our model is that the spinors live in the complex projective space $C P^{d-1}$, which supports exact multi-Skyrmion solutions irrespective of the value of $d$, and, most crucially, the assumption of large value of spin stiffness.

Our programme consists of two parts. First, we derive a set of largely analytical results on the case of Skyrmion crystals which are described locally by $S U(d)$ spinors. We find that macroscopic degeneracy, present in the noninteracting case [12], is lifted in favour of a hexagonal SC with the amplitude of charge density modulations decaying exponentially with $d$. The excitation spectrum, obtained by studying small perturbation of the crystal, exhausts the number of gapless modes consistent with the original degeneracy. Here, $d^{2}-1$ of these modes correspond to generators of the $S U(d)$ Lie algebra, in accordance with the Goldstone theorem, supplemented by a magnetophonon excitation, appearing as a result of broken translational invariance. The magnetophonon has a dispersion reminiscent of a 2D Wigner crystal in a magnetic field [13].

Second, as an application of our theory, we consider explicitly a model of multi-component quantum Hall ferromagnet, motivated by experiments on semiconductor quantum wells. The effects of anisotropies, present in these systems, which break $S U(d)$ symmetry, are studied. With increasing $d$, the number of possible types of anisotropies grows quickly along

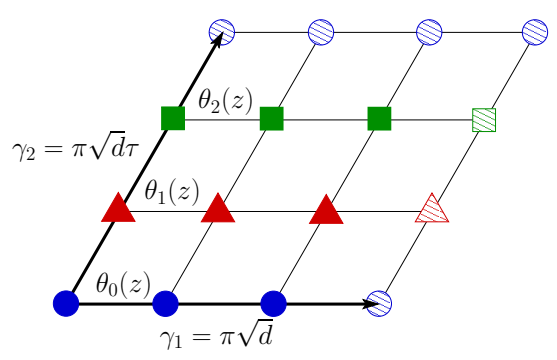

FIG. 1: (Color online). Positions of zeros of the basis theta-functions $\theta_{p}(z)$ within a single unit cell of a Skyrmion lattice in the $S U(3)$ case. The lattice vectors are indicated by arrows. 
with the $d^{2}-1$ generators of $S U(d)$, and we concentrate on the simplest nontrivial, yet instructive, and hitherto unstudied case of $S U(3)$ Skyrmions. These are of experimental interest for quantum wells grown in a [111] direction, in particular a nascent effort on AlAs, where one finds the appearance of three almost degenerate valleys [8]. The latter are subject to a 'nematic' anisotropy recently discussed in the context of $S U(2)$ valley degrees of freedom [14], which we generalise to the case of an arbitrary number of valleys. We show how this anisotropy changes the nature of the crystalline ground state by combining spontaneous symmetry breaking with the externally imposed one, leading to a collection of different crystal structures. We also determine the gaps appearing in the lowenergy excitation spectrum.

The remainder of the paper is organised as follows. First, we introduce the $C P^{d-1}$ model describing spatial variations of internal degrees of freedom, taking into account interactions between electrons. An exact HF ground state in terms of spinors living in an analytic subspace is presented. Starting from this state we derive linearized equations of motion describing excitations of Skyrmion lattices. A generalization of Bloch theorem leads, in the isotropic case, to a reduction of a system of $2 d^{2}$ equations into $d^{2}$ decoupled pairs of linear equations, which analytical solution provides the spectrum together with the eigenfunctions of the excitations. Next, we consider a model of semiconductor, possessing three degenerate valleys, which takes into account the 'nematic' anisotropy, and present results for the phase diagram and excitations.

The model. We consider a quantum Hall ferromagnet at filling factor $\nu \sim 1$ with internal degrees of freedom described by a $d$-component spinor $w_{n}(\mathbf{r})$, which corresponds to the Slater determinant state $|\Psi\rangle=\Pi_{\mathbf{r}}\left[\sum_{n=1}^{d} w_{n}(\mathbf{r}) \hat{c}_{n}^{\dagger}(\mathbf{r})\right]|0\rangle$, where the spinor is normalized as $\sum_{n=1}^{d}\left|w_{n}(\mathbf{r})\right|^{2}=1$ at every point $\mathbf{r}$, $\hat{c}_{n}^{\dagger}(\mathbf{r})$ creates an electron in the $n$-th spin component in the lowest Landau level (LLL) eigenstate with position $\mathbf{r}$. The latter are given in the coherent state representation, and $|0\rangle$ is the electron vacuum. For example, in the case of a bilayer quantum Hall system, components of the spinor correspond to layer and spin indices, and in graphene to spin and valley. The Hamiltonian of the system is given by two contributions $H=H_{\mathrm{CP}}+H_{\text {int }}$, where $H_{\mathrm{CP}}$ is proportional to the spin stiffness $\rho_{S}$, and $H_{\text {int }}$ describes Coulomb interactions. A generic form of $H_{\mathrm{CP}}$, invariant under $S U(d)$ rotation and containing only first order gradients reads [7, 15]

$$
H_{\mathrm{CP}}=2 \rho_{S} \int d^{2} \mathbf{r}\left[\frac{(\nabla w, \nabla w)}{(w, w)}-\frac{(w, \nabla w)(\nabla w, w)}{(w, w)^{2}}\right],
$$

where the integral is taken over the two dimensional plane, and the scalar product is defined as $(a, b) \equiv \sum_{n} a_{n}^{*}(\mathbf{r}) b_{n}(\mathbf{r})$. The Hamiltonian has a local "gauge" symmetry, in other words, multiplying a spinor by an arbitrary function, $\tilde{w}_{a}(\mathbf{r})=$ $h(\mathbf{r}) w_{a}(\mathbf{r})$, does not affect the energy. The electron interactions with a potential $V\left(\mathbf{r}-\mathbf{r}^{\prime}\right)$ are described by

$$
H_{\mathrm{int}}=\frac{1}{2} \int d^{2} \mathbf{r} d^{2} \mathbf{r}^{\prime} V\left(\mathbf{r}-\mathbf{r}^{\prime}\right) Q(\mathbf{r}) Q\left(\mathbf{r}^{\prime}\right),
$$

in the lowest order of the gradient expansion. Here $Q(\mathbf{r})$ is the topological charge density, which is proportional to the electronic charge density in the quantum Hall effect [1, 15], and is related to a Pontryagin index of the normalized spinor field [12]. In the following we consider textures in the form of Skyrmion crystals and fix the topological charge, given by the integral of $Q(\mathbf{r})$ inside the unit cell (u.c.), to $2 \pi d$. The two lattice vectors of the unit cell, which define a parallelogram with opening angle $\phi$, can be chosen as $\gamma_{1}=\pi \sqrt{d}$ and $\gamma_{2}=\pi \sqrt{d} \tau$, where $\tau=e^{i \phi}$ (real/imaginary parts of $\gamma_{1,2}$ correspond to $x, y$ components of the vectors). We measure the lengths in units of $2 l_{B} / \sqrt{|\nu-1| \operatorname{Im} \tau}$.

The energy $H_{\mathrm{CP}}$ is minimized by spinors, with entries analytic functions of $z=x+i y$, whose number of zeros inside the u.c. fixes the topological charge sector. Without interactions there is a macroscopic degeneracy coming from the arbitrariness in the choice of positions for these zeros. In presence of $H_{\text {int }}$ this degeneracy is lifted, which can lead to formation of a Skyrmion crystal. It is impossible to write an analytic expression for the ground state in the interacting case, thus we propose a variational approach in which the trial state is still expressed in terms of analytic functions $w_{a}(z)$. Although the Euler-Lagrange equations, derived from $H_{\mathrm{CP}}$, are highly nonlinear, our procedure is reminiscent of the LLL projection, familiar from the theory of Abrikosov vortex lattices and the QHE physics. Our conjecture is that the zero modes of the Hessian of $H_{\mathrm{CP}}$, spanning the analytic subspace, are separated from the higher (non-analytic) ones by a spectral gap of the order $\rho_{S}$, because the average topological charge density, analogous to a magnetic field strength, is independent of $d$. Further, the energy scale associated with the Coulomb interaction is $\rho_{S} \sqrt{n_{S}} l_{B}$, where $n_{S}$ is the Skyrmion density and $l_{B}$ describes the magnetic length. This scale is smaller than $\rho_{s}$ provided that $n_{S} l_{B}^{2} \ll 1$, i.e. if the filling factor remains close to one. This justifies our variational treatment.

The method. We choose a basis of analytic functions defined by their quasi-periodicity $\theta(z+\gamma)=e^{a_{\gamma} z+b_{\gamma}} \theta(z), \gamma=$ $n_{1} \gamma_{1}+n_{2} \gamma_{2}$, where $a_{\gamma}, b_{\gamma}$ are complex numbers and $n_{1,2} \in$ $Z$. Using the properties of these functions under translations $\hat{T}_{\gamma_{2} / d} \theta_{p}(z)=e^{-i \pi \tau(d+1) / d} \theta_{p+1}(z)$ and $\hat{T}_{\gamma_{1} / d} \theta_{p}(z)=$ $e^{2 i \pi p / d} \theta_{p}(z)$, we obtain the basis of linearly-independent theta-functions with a given topological charge inside the u.c.

$$
\theta_{p}(z)=\sum_{n=-\infty}^{\infty} e^{i \pi \tau d\left(n-\frac{p}{d}\right)\left(n-1-\frac{p}{d}\right)+2 i \sqrt{d}\left(n-\frac{p}{d}\right) z},
$$

where $p$ runs from 0 to $d-1$, in agreement with RiemannRoch theorem [16]. From the numerical minimization procedure, which was further supported by a linear stability analysis, we find that the energy minimum is achieved for a spinor formed from basis functions with equal amplitudes (up to a global $S U(d)$ rotation) $w_{a}(z)=\left(\theta_{0}, \ldots, \theta_{d-1}\right)$, and $\tau=e^{i \pi / 3}$. This corresponds to a charge distribution with the full symmetries of a hexagonal lattice, although the individual spinor components possess a lower symmetry.

In order to calculate the excitations spectra of the SC we use the basis formed from a set of quasi-periodic analytic func- 
tions $\chi_{p \mathbf{k}}(z)$ obtained from $\theta_{p}(z)$ by translations by vector $\mathbf{k}$ with components $2 \pi m_{1} / N_{1}$ and $2 \pi m_{2} / N_{2}$, where $m_{1}, m_{2} \in$ $Z$ and $N_{1}$ and $N_{2}$ is the number of unit cells on a torus. An arbitrary analytic spinor can be written in terms of $\chi_{p \mathbf{k}}(z)$ as

$$
w_{a}(z)=\sum_{\mathbf{k} \in \mathbf{B Z}} \sum_{b=1}^{d} \mathcal{U}_{a b}(\mathbf{k}) \chi_{b \mathbf{k}}(z), \quad a=0 . . d-1
$$

see [17] and a related work [18], where an analogous approach was formulated to a $U(1)$ case for the problem of vortex lattices in Bose-Einstein condensates. Here we introduce arbitrary complex square matrices $\mathcal{U}$ of coefficients with linear dimension $d$. We consider time-dependent HF, with dynamics generated by a Lagrangian given as a sum of the energetic term $H_{\text {int }}$ and a Berry's phase term, which is first order in time derivatives of the spinor components. Variation of the Lagrangian with respect to parameters $\mathcal{U}_{a b}(\mathbf{k})$ gives a system of coupled equations describing the motion of a Skyrmion lattice, constrained to the analytic subspace

$$
\begin{aligned}
\int d^{2} \mathbf{r} \frac{\chi_{b \mathbf{k}}^{*}(z)}{(w, w)}\left\{i \frac{\partial w_{a}}{\partial t}-i \frac{\left(w, \partial_{t} w\right)}{(w, w)} w_{a}(z)\right. \\
\left.\quad-4 w_{a}(z) \int \Delta_{\mathbf{r}} V\left(\mathbf{r}-\mathbf{r}^{\prime}\right) Q\left(\mathbf{r}^{\prime}\right) d^{2} \mathbf{r}^{\prime}\right\}=0 .
\end{aligned}
$$

Equation (5) represents one of the main results of our paper.

LSWT and Bloch theorem. The system of equations (5) is very general and is not amenable to analytic treatment, mostly because the velocity field $\partial w_{a} / \partial t$ is not explicit. However, due to the high degree of symmetry of our reference SC state, it is possible to obtain full analytical results at arbitrary $d$ for small perturbations of the lattice, i.e. for linearized equations of motion. The matrices of coefficients now can be written as a sum $\mathcal{U}_{a b}(\mathbf{k})=\delta_{a b} \delta_{\mathbf{k}, 0}+u_{a b}(\mathbf{k})$, where $u_{a b}(\mathbf{k})$ are small. The quasi-periodicity of theta-functions and the periodicity

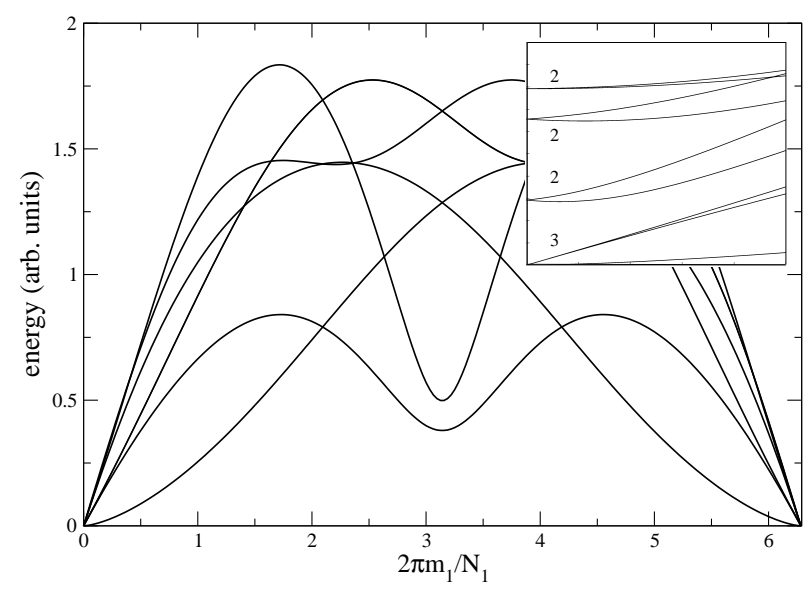

FIG. 2: Excitation spectra of a $S U(3)$ Skyrmion lattice, in the case of Coulomb interactions, along a diagonal direction in the Brillouin zone, $k_{1}=k_{2}$. The lowest lying mode at small $k_{1}$ is a magnetophonon. All remaining modes are linear gapless acoustic excitations. (inset) appearance of gaps in the $S U(3)$ case at finite anisotropy strength. (with elementary periods $\gamma_{1} / d$ and $\gamma_{2} / d$ ) of the topological charge density of the SC leads to a generalized Bloch theorem, and the system of equations fully decouples into $d^{2}$ pairs of linear equations. These are readily solved, resulting e.g. in the excitation spectrum for a $S U(3)$ case presented in Fig. 2.

Results. The magnetophonon mode at small energies has a dispersion $|\mathbf{k}|^{\alpha}$, with $\alpha \sim 3 / 2$ in the case of Coulomb interactions, like the dispersion of a 2D Wigner crystal in a magnetic field. For a short-range interaction $\alpha \sim 2$, while the spectrum is linear at small energies, $\alpha \sim 1$, for logarithmic (2D Coulomb) interactions. In the large- $d$ limit, topological charge density variations of a SC have an exponentially small amplitude, for example for a square lattice we find

$Q(\mathbf{r})=2 / \pi-4 d e^{-\frac{\pi d}{2}}[\cos (2 \sqrt{d} x)+\cos (2 \sqrt{d} y)]+O\left(e^{-\pi d}\right)$,

Coulomb interactions suppress charge density fluctuations, and, for a large number of components, it becomes possible to arrange for a nearly-uniform twist of the spinor. Interestingly, similar questions arise in the studies of $S U(N)$ superconductors [19].

$S U(3)$ case. Let us discuss application of our general methods to a potentially realizable case of Skyrmion crystals in AlAs quantum wells, where the spin degree of freedom is quenched due to applied magnetic field, and the valleydegeneracy leads to the three-component spinors with coefficients $\alpha_{i}$, which we describe in zeroth order by a $S U(3)$ symmetric theory. Effective mass anisotropy within the valleys with relative angle $\varphi$ generates a symmetry-breaking term in the Hamiltonian describing a 'nematic' anisotropy

$$
H_{\mathrm{N}}=-\Delta_{0} c_{1}+2 \Delta_{0} \varkappa \sum_{i \neq j}\left|\alpha_{i}\right|^{2}\left|\alpha_{j}\right|^{2}
$$

where $\Delta_{0}=\sqrt{\pi / 8} e^{2} / \varepsilon l_{B}$ is the exchange energy in the isotropic case, and $l_{B}=\sqrt{\hbar c / e B}$ is the magnetic length. With $K(x)$ denoting the complete elliptic integral of the first kind, and $\varkappa=\left(c_{1}-c_{2}\right) / 2$, the coefficients are given by

$$
c_{1}=\frac{2}{\pi} \frac{K\left(1-1 / \lambda^{2}\right)}{\sqrt{\lambda}}, \quad c_{2}=\frac{2}{\pi} \frac{K\left(1-b^{2} / a^{2}\right)}{a},
$$

where $a^{2}=\lambda \cos ^{2} \frac{1}{2} \varphi+\lambda^{-1} \sin ^{2} \frac{1}{2} \varphi$ and $b^{2}=\lambda \sin ^{2} \frac{1}{2} \varphi+$ $\lambda^{-1} \cos ^{2} \frac{1}{2} \varphi$, and $\lambda=\left(m_{x} / m_{y}\right)^{1 / 2}$ stands for the anisotropy parameter. Notice that $\varkappa \geq 0$, describing repulsive interaction between the different components $\alpha_{i}$ of the spinor. This reduces to the form derived in [14] for the $S U(2)$ case. In the isotropic limit, $m_{x}=m_{y}$, the ground state, for a given filling factor, is a triangular Skyrmion lattice, which transforms into a square one with increasing value of the anisotropy strength, see Fig. 3. This transition is due to the relative scaling of Coulomb and nematic energies with $\operatorname{Im} \tau$. We do not find the nematic phase transition of [14] even at large values of anisotropy strength. Note that although valley polarization is favoured by the anisotropic term, its Coulomb energy cost turns out to be so large that the different spinor amplitudes 

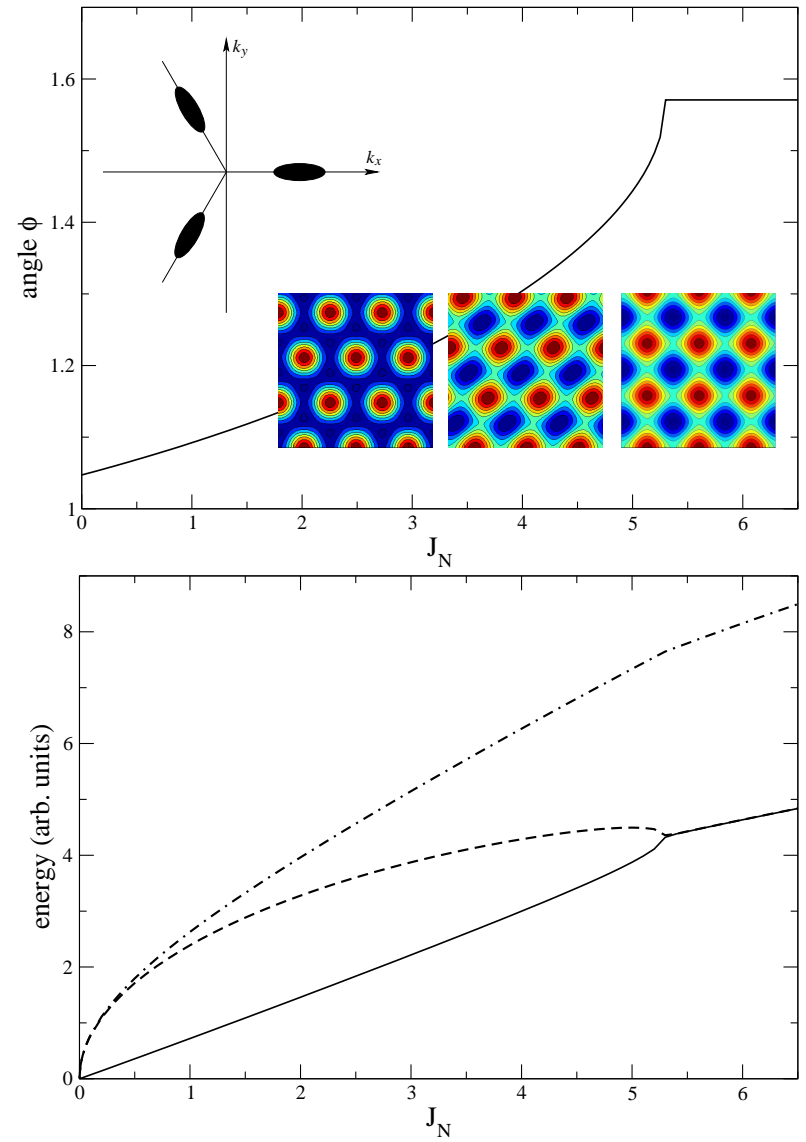

FIG. 3: a) Angle $\phi$ in the $S U(3)$ case as a function of nematic anisotropy strength. The crystal is hexagonal in the isotropic case and becomes square at large $J_{N}=\varkappa \exp (\pi d / 2) / \pi \sqrt{2 \pi}|\nu-1|^{3 / 2}$. b) Scaling of gaps with nematic anisotropy strength. The first gap shows linear behavior, while the other two increase as a square root. (upper inset of Fig.a) Schematic picture of the valleys in AlAs quantum wells in the [111] direction showing the three-fold valley degeneracy. The effective mass within each valley is anisotropic with $m_{x} / m_{y} \approx 4$. (lower inset of Fig.a) topological charge densities of a Skyrmion crystal from weak to strong anisotropy.

in our variational ground-state remain equal, independently of the value of $\varkappa$.

Finite gaps appear in the spectrum in presence of anisotropy. The first three modes remain gapless; they correspond to magnetophonon and to the two remaining continuous symmetry generators associated with a two-dimensional Cartan subgroup of $S U(3)$. The other six modes are now gapped, so that the collective spectrum forms the pattern shown in Fig.3. The first two gaps scale linearly with the anisotropy strengths, while the behavior is $\sim \sqrt{J_{N}}$ for the other four.

Energy scales. In the SU(3) valley-degenerate case we obtain at small anisotropies $\mathcal{A}_{N} \equiv \Delta_{0} \varkappa / 2 \pi l_{B}^{2} \sim \frac{9}{128} \frac{\Delta_{0}}{2 \pi l_{B}^{2}}(\lambda-$ $1)^{2}$, and $\rho_{S}=e^{2} / 16 \sqrt{2 \pi} \varepsilon l_{B} \sim 5.2 \mathrm{~K}$, with $\Delta_{0} \sim 131 \mathrm{~K}$. The anisotropy energy $\Delta_{0} \varkappa \sim 2.5 \mathrm{~K}$ is much smaller than the spin stiffness scale $4 \pi \rho_{S} \sim 65 \mathrm{~K}$, where we assumed $\varepsilon=$ $10 \varepsilon_{0}$ for AlAs at $\nu=1$ and electron density $2.5 \times 10^{11} \mathrm{~cm}^{-1}$
[20]. The characteristic Coulomb energy is $\sim \rho_{S}|\nu-1|^{1 / 2}$ and can be made small compared to the stiffness by changing the filling factor. For relatively small values of $|\nu-1| \sim 0.1$ we estimate the value of dimensionless parameter $J_{N} \sim 13.5$ which corresponds to a square lattice, see Fig.3.

Conclusions. We have studied $S U(d)$ quantum Hall ferromagnets with finite density of topological defects (Skyrmions). In presence of Coulomb interactions the ground state of the system is a triangular Skyrmion lattice, which breaks all the symmetries of the internal $S U(d)$, as well as translational symmetry, generating $d^{2}$ Goldstone modes, $d^{2}-1$ of which correspond to breaking of the $S U(d)$, and the remaining one being a magnetophonon mode. We have explored the phase diagram of a $S U(3)$ QHE ferromagnet, which is expected to be relevant to semiconductor nanostructures with valley-degeneracies [8]. The lattice tilts continuously as a function of anisotropy strength and becomes square through a phase transition at a critical anisotropy value. In this case, three of the excitation branches remain gapless, while others acquire gaps exhibiting different scaling with anisotropy strength.

It would be interesting to extend our theory to the case of large, but finite, spin-stiffness, and to derive an effective sigma-model for the Skyrmion lattice in the projected subspace. The difficulty which one immediately faces is that long-wavelength $S U(N)$ rotations are not preserved under projection onto the analytic subspace. Another important question is related to entanglement of internal degrees of freedom, as studied for simple Skyrmions structures in [21].

Acknowledgements. We are grateful to J. T. Chalker for insightful comments. D. K. acknowledges hospitality of the Theoretical physics department at Oxford.

[1] S. L. Sondhi, A. Karlhede, and S. A. Kivelson, E. H. Rezayi, Phys. Rev. B 47, 16419 (1993).

[2] X. Z. Yu, Y. Onose, N. Kanazawa, J. H. Park, J. H. Han, Y. Matsui, N. Nagaosa and Y. Tokura, Nature 465, 901 (2010); S. Mühlbauer, B. Binz, F. Jonietz, C. Pfleiderer, A. Rosch, A. Neubauer, R. Georgii, P. Böni, Science 323, 915 (2009).

[3] A. Bogdanov and A. Hubert, Journ. of Magn. and Magn. Mater. 138, 255 (1994); A. Bogdanov, and U. Rößler, Phys. Rev. Lett. 87, 037203 (2001). J. H. Han, J. Zang, Z. Yang, J.-H. Park, and N. Nagaosa, Phys. Rev. B 82, 094429 (2010).

[4] R. W. Cherng and E. Demler, Phys. Rev. A 83, 053613 (2011); Phys. Rev. A 83, 053614 (2011).

[5] R. Tycko, S. E. Barrett, G. Dabbagh, L. N. Pfeiffer, and K. W. West, Science 268, 1460 (1995).

[6] S. Q. Murphy, J. P. Eisenstein, G. S. Boebinger, L. N. Pfeiffer, and K. W. West, Phys. Rev. Lett. 72, 728 (1994).

[7] S. Girvin, "The Quantum Hall Effect: Novel Excitations and Broken Symmetries", arXiv:cond-mat/9907002

[8] S. Prabhu-Gaunkar, S. Birner, S. Dasgupta, C. Knaak, and M. Grayson, Phys. Rev. B 84, 125319 (2011); K. Eng, R. N. McFarland, B. E. Kane, Phys. Rev. Lett. 99, 016801 (2007); F. Herzog, M. Bichler, G. Koblmüller, S. Prabhu-Gaunkar, W. Zhou, M. Grayson, Appl. Phys. Lett. 100, 192106 (2012). 
[9] Y. Sakurai and D. Yoshioka, Phys. Rev. B 85, 045108 (2012); M. O. Goerbig, Rev. Mod. Phys. 83, 1193 (2011)

[10] I. Klebanov, Nuclear Physics B 262, 133, (1985); T. H. R. Skyrme, Proc. Roy. Soc. 260, 127 (1961).

[11] R. Côté, D. B. Boisvert, J. Bourassa, M. Boissonneault, and H. A. Fertig, Phys. Rev. B 76, 125320 (2007).

[12] R. Rajaraman, Solitons and Instantons North-Holland, Amsterdam, 1982; A. M. Polyakov, Gauge Fields and Strings, Harwood Academic Publishers, 1987; Z. F. Ezawa, Quantum Hall Effects: Field Theoretical Approach and Related Topics, World Scientific, 2008.

[13] H. Fukuyama, Solid State Comm., v. 17, 10, 1323 (1975); R. Côté and A. H. MacDonald, Phys. Rev. B 44, 8759 (1991); A. G. Green, I. I. Kogan and A. M. Tsvelik, Phys. Rev. B 54, 16838 (1996).

[14] D. A. Abanin, S. A. Parameswaran, S. A. Kivelson, S. L. Sondhi, Phys. Rev. B 82, 035428 (2010).
[15] K. Moon, H. Mori, Kun Yang, S. M. Girvin, A. H. MacDonald, L. Zheng, D. Yoshioka, Shou-Cheng Zhang, Phys. Rev. B 51, 5138 (1995).

[16] O. Debarre, "Complex tori and Abelian varieties", American Mathematical Society, Texts and Monographs, (1999).

[17] F. D. M. Haldane, E. H. Rezayi, Phys. Rev. B. 31, 2529 (1985); G. Eilenberger, Phys. Rev. 164, 628 (1967).

[18] S. I. Matveenko and G. V. Shlyapnikov, Phys. Rev. A 83, 033604 (2011).

[19] M. A. Moore, T. J. Newman, A. J. Bray, and S-K. Chin, Phys. Rev. B 58, 936 (1998).

[20] Y. P. Shkolnikov, S. Misra, N. C. Bishop, E. P. De Poortere, and M. Shayegan, Phys. Rev. Lett. 95, 066809 (2005).

[21] B. Douçot, M. O. Goerbig, P. Lederer, and R. Moessner, Phys. Rev. B 78, 195327 (2008). 